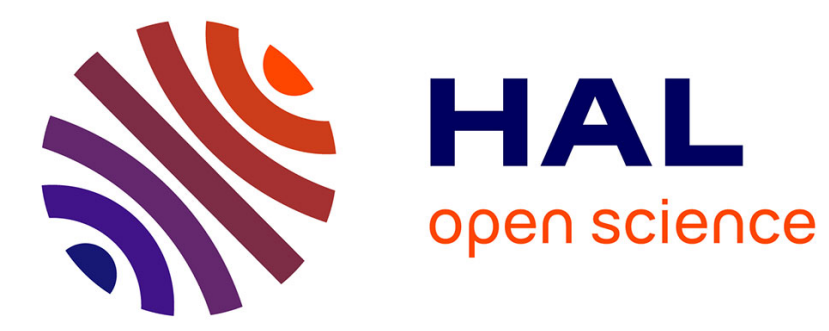

\title{
Convex Storage Loss Modeling for Optimal Energy Management \\ Pierre Haessig
}

\section{To cite this version:}

Pierre Haessig. Convex Storage Loss Modeling for Optimal Energy Management. PowerTech 2021 (on line), Jun 2021, Madrid, Spain. 10.1109/PowerTech46648.2021.9494786 · hal-03032241v2

\section{HAL Id: hal-03032241 \\ https://hal.science/hal-03032241v2}

Submitted on 14 Apr 2021

HAL is a multi-disciplinary open access archive for the deposit and dissemination of scientific research documents, whether they are published or not. The documents may come from teaching and research institutions in France or abroad, or from public or private research centers.
L'archive ouverte pluridisciplinaire HAL, est destinée au dépôt et à la diffusion de documents scientifiques de niveau recherche, publiés ou non, émanant des établissements d'enseignement et de recherche français ou étrangers, des laboratoires publics ou privés. 
Copyright warning: this manuscript was accepted at the IEEE PowerTech 2021 conference. Copyright belongs to IEEE. Also, a few extra paragraphs and one figure that had to be trimmed to match the 6-page submission limit are added back and marked in blue.

\title{
Convex Storage Loss Modeling for Optimal Energy Management
}

\author{
Pierre Haessig \\ IETR \\ CentraleSupélec \\ Rennes, France \\ pierre.haessig@centralesupelec.fr
}

\begin{abstract}
Models of energy storage systems used for optimal energy management commonly feature unsophisticated loss expressions (lossless or constant efficiency). Preserving the tractability (i.e. convexity) of the optimization motivates this simpleness, at the expense of physical realism. Relaxing the storage loss expression from an equality constraint to an inequality allows using more complex and physically realistic models while preserving convexity. Although this relaxation has been used quite casually for a decade in varied energy management applications (power systems dispatch, electric vehicles), only few articles studied this technique for itself. To increase the awareness of loss relaxation, along with its limitations, we depict a unified view of relaxed storage loss models. Also, we propose the "convex monomial loss model", one continuously parametrized family of nonlinear convex expressions which contains all classical loss models and unlocks new possibilities for loss dependency in storage power and energy level. This is one step to reunite piecewise linear and nonlinear models which are generally studied separately. Finally, we compare the effect of these varied loss models on the optimal charge/discharge profile of a storage in an elementary energy management application.
\end{abstract}

Index Terms - convex optimization, energy management, energy storage, storage losses, storage models

\section{INTRODUCTION}

Energy storage systems (ESSs), in particular batteries, are being used in a large and growing number of applications, down from small portable devices up to large power systems. Energy management is the specific name for the control of the power flows in a system which includes one or more energy storages, to achieve desired objectives like energy shifting, power shaving, backup...

Energy management can sometimes be achieved by simple rules, but many storage applications have complex enough requirements to justify using mathematical optimization tools instead. A large subset of optimization-based control methods, like Model Predictive Control, require solving optimization problems online. This necessitates solving those optimizations quickly and reliably and this reliability depends essentially on the class of optimization problem being solved.

This need pushes control designers to formulate their problems as Convex Programs (CPs), because it is a wide class of problems for which reliable solvers exist, in particular for the common Linear and Quadratic subclasses (LPs and QPs) [1]. True, there also exist popular and useful classes of nonconvex problems, mainly the previous ones expanded with some integrality constraints. The resulting problems (MILP, MIQP...) benefit from powerful solvers, but fundamentally don't share the nice convergence properties of convex ones. This justifies the search for convex formulations of energy management problems, if physically possible.

As detailed in section II-A, for a model-based energy management problem to be convex, storage loss expression must be linear. This is very restrictive (see II-C) and means that in many applications, storage losses are simplified (e.g. [2]) or fully neglected just to satisfy convexity and not for physical reasons.

To overcome this limitation, befitting constraint relaxation schemes have been used. Indeed, relaxation is a typical method to "convexify" a variety of problems, for example Optimal Power Flows (e.g. [3]). For energy management, these methods all come to relaxing the storage loss expression from an equality constraint (losses $=$ some expression) to an inequality (losses $\geq$ some expression) [4]. The rationale is that, at the optimum, no energy should be wasted, so the inequality should be tight. We call this the "positive energy price argument" and come back to it further down. Switching to an inequality allows using any nonlinear convex expressions for losses, while keeping the resulting energy management problem convex. Then, the choice of loss expression depends on both the application and the type of storage technology being modeled.

The most popular model (beyond the lossless case mentioned above) is, by far, the piecewise-linear-in- $P$ expression (hereafter PWL-in- $P$, e.g. $\propto|P|$ ), which is generally transcribed using a constant charging/discharging efficiency $(\eta<1)$ (see [2], [5]-[8] for a tiny subset of use cases). This is a physics-free and generic storage model which is typically formulated as a LP, while it is a non-convex MILP without the relaxation (see III-A). The recent work of GonzalezCastellanos et al. [9], although being a much wider effort of convex battery modeling (not just losses), also yields a closely related model (PWL-in- $P$ with a linear effect of the energy level).

Inspired by resistive heating, a quadratic-in- $P$ model (i.e. $\propto P^{2}$ ) is more physical for electrochemical storage technologies. For optimal energy management, these devices can be represented by the circuit model visible on the right-hand part of Fig. 1. Convex optimization of this model was proposed by Murgovski et al. [4] in a battery intensive application 


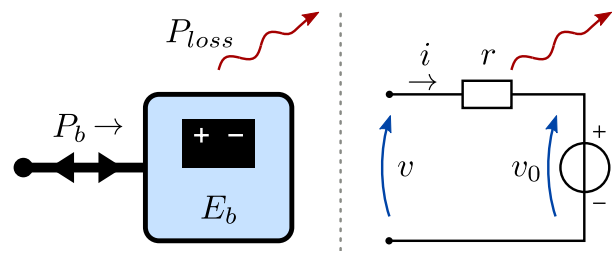

Fig. 1. Energy storage models. On the left: energy based model which is ubiquitous in energy management optimization. On the right: circuit based model which is more physical for electrochemical devices ( $v_{0}$ and $r$ are often state dependent). Conversion between the two is given Section III-B.

(Hybrid Electric Vehicle) along with a detailed, first of its kind, discussion on the "convexification" the circuit model. Also, it's maybe because quadratic terms often appears in power flow equations that we found a resembling model in a distribution grid control application [10]. Section III-B is devoted to this model.

Now, the most complex and genuinely nonlinear loss expression we found is in the follow-up work of Murgovski et al. [11] and subsequently used by Pinto et al. [12]. Their $P^{2} / E$ loss expression (with $E$ being the storage energy level) allows modeling storage technologies for which the open circuit voltage ( $v_{0}$ on Fig. 1$)$ varies with the energy level $E$. We discuss and generalize this in section IV.

This literature review shows that the practice of relaxing storage loss expressions exist. Still, it is not ubiquitous (see e.g. [13] for a MILP energy management where the integrality constraints associated to the storage model may have been relaxed, although it may not be the case for other components).

Also, this relaxation is considered as natural enough that often the "positive price argument" is only lightly mentioned, if not omitted, although it cannot apply sometimes. Indeed the relaxation can fail in the sense that the relaxed loss inequality can be not tight so that more energy is wasted than prescribed by the loss expression. A notable exception to this casualness is Almassalkhi et al. [5, App. A] which not only explain the argument, but also give a worst-case bound for when the relaxation is not tight. Also, they propose a heuristic algorithm to fix this. Alternatively, Z. Li et al. [14] and Duan et al. [15] identified mathematically sufficient conditions for the relaxation to be exact. Still, these conditions do not always apply [8]. Recently, Arroyo et al. [16] refuted two Transactions articles which used too simple conditions! We thus feel the need to shed one more light on these useful storage loss relaxation techniques, while carefully referencing the limitations.

Finally, the literature shows a complete split between the popular physics-free PWL-in- $P$ loss model and the more elaborate $P^{2}$ or $P^{2} / E$ models. However, when searching for a convex model fitted to experimental data, it may be useful to have a continuously parametrized family of loss expressions. Thus, it is our core contribution in this paper to:

1) Describe the relaxation of storage losses in a unified way which handles the various existing loss models

2) Provide a simple continuous family of nonlinear convex loss expressions which can depend on both storage power and energy level. This family contains existing models (PWL, quadratic, $P^{2} / E$ ) as special cases.

The unified description of loss models is covered in sections II and III, while our new "convex monomial loss model" is described in IV. Finally, in section V we create an elementary energy management application with one energy storage. We use this example to compare the effect of the varied loss models on the optimal charge/discharge profile of the storage.

\section{Convex Storage Modeling WITH LOSS RELAXATION}

\section{A. Recall of Convexity}

We recall the conditions for an optimization problem to be convex (see [4, §2] or textbook [1] for more background). Optimization problems, as often used in energy management, can be formalized as:

$\min _{x \in \mathbb{R}^{n}} f(x)$, such that $g_{i}(x) \leq 0, h_{j}(x)=0(i, j=1,2 \ldots)$

For this problem to be convex, the cost function $f$ must be convex and so must be the set of feasible solutions $\left\{x\right.$ such that $g_{i}(x) \leq 0$ and $\left.h_{j}(x)=0, \forall i, j\right\}$. The latter implies that each inequality function $g_{i}$ must be convex and each equality function $h_{j}$ must be linear. This will be crucial to our discussion, because few practical storage loss expressions are linear.

\section{B. Generic Storage Model}

We start with the following generic energy storage model:

$$
E_{b}(k+1)=E_{b}(k)+\left(P_{b}(k)-P_{\text {loss }}\right) \Delta_{t}
$$

It is expressed in discrete time (instant $k$, timestep $\Delta_{t}$ ) and describes how the stored energy $E_{b}$ evolves with the storage power $P_{b}$ (in receptor convention) including losses $P_{\text {loss. }}$. Because all variables are discrete signals, we drop below the time index $k$ for relations applying for all $k$. This is the most common type of model for energy management optimization, while electrochemical models are more often expressed with charge, current and voltages (see Fig. 1). The conversion between the two is discussed further (section III-B).

The stored energy is bounded, which is typically modeled with:

$$
0 \leq E_{b} \leq E_{\text {rated }}
$$

where $E_{\text {rated }}$ is the maximum usable energy of the system. From it, one can define the state of energy $S o E=$ $E_{b} / E_{\text {rated }} \in[0,1]$. Storage power is also bounded by

$$
-P_{\text {rated }}^{-} \leq P_{b} \leq P_{\text {rated }}^{+}
$$

Constraint (3) allows asymmetrical charge and discharge rate limits. A rich extension is to consider a more general convex set for the pair of variables $\left(P_{b}, E_{b}\right)$, instead of the rectangular box defined by (2)-(3), see for example [9], [11].

Equations (1)-(3) describe a convex set of constraints to be embedded in a larger optimization problem involving $E_{b}(k), P_{b}(k), P_{\text {loss }}(k)(k=1 \ldots K)$ as decision variables. 
However, storage dynamics (1) is generic because the storage losses $P_{\text {loss }}$ have yet to be specified. So the convexity of the complete problem depends on the loss expression.

\section{The Limited Usability of Linear Loss Expressions}

The simplest convex model is the lossless storage

$$
P_{\text {loss }}=0
$$

which, of course, in practice is used to simplify (1).

The only convex generalization of this, assuming losses depend on power and state of energy, is the linear expression

$$
P_{\text {loss }}=p_{0}+c_{P} P_{b}+c_{E} E_{b} .
$$

Parameter $p_{0}$ models a constant self-discharge, while coefficient $c_{E}$ modulates the discharge with the state of energy. Both are physically meaningful and have some popularity (e.g. [2]). In contrast, we have never seen the use of the $c_{P}$ coefficient. Indeed, since power $P_{b}$ can take both signs, there would be some physical contradiction in assuming that losses may be reduced for large charge $\left(c_{P}<0\right)$ or discharge $\left(c_{P}>0\right)$ rates.

\section{Generic Relaxation of Storage Losses}

The limited physical applicability of the linear loss model yielded befitting relaxation techniques (e.g. [4], [5], [14]). The generic formulation, expressed again here with a dependency on storage power and energy ${ }^{1}$, is:

$$
P_{\text {loss }} \geq g\left(P_{b}, E_{b}\right)
$$

where $g$ means here any convex function. This formulation rests on three principles, sorted by decreasing order of givenness:

1) Mathematical fact: with $g$ convex, (6) indeed defines a convex inequality constraints as in II-A

2) Physical observation: the fact that storage losses generally increase for large positive and negative values of power $P_{b}$ and are minimal for $P_{b}=0$ motivates using a function $g$ convex in $P_{b}$ (e.g. quadratic, absolute value...)

3) Application-dependent hypothesis: most optimal energy management problems embed the property that the optimum is reached by minimizing wasted energy, so that inequality (6) should be tight at the solution. We call this the "positive price argument", which is often mentioned in various ways (e.g. [6], [10], [12]).

\section{Remarks:}

1) We know no principle similar to 2) to justify the convexity of $g$ in $E_{b}$ nor its joint convexity. Therefore, these hypotheses appear only as useful mathematical tricks which applicability needs to be proved. This applicability question would be the same if losses should depend on more variables.

2) Under principle 3) that losses should be minimized, the loss function $g$ does not need to be an explicit expression. Instead it can be the solution of a convex

\footnotetext{
${ }^{1}$ Two extra meaningful dependencies for losses are temperature and aging.
}

minimization subproblem which can be embedded in the global problem. See examples below.

3) Some precise sufficient conditions have been identified to replace principle 3), but they are not necessary, cannot always be checked ex-ante and are application-specific (see [8], [14], [15]).

4) In the literature, the relaxation we introduce in (6) is more commonly written by inserting the loss expression directly in the storage dynamics (1) and relaxing the equality as $E(k+1) \leq E(k)+(\ldots)$. It is equivalent and more compact, but we carve out the losses here to ease the discussion on their expression.

\section{Existing Convex Loss Models}

There are two commonly used convex expressions that can play the role of $g$ in (6).

\section{A. Piecewise-Linear-in-P Loss Model}

The most common loss model is the PWL-in- $P$ expression (e.g. used in [2], [5]-[8], but also in many other studies):

$$
g\left(P_{b}, E_{b}\right)=c_{+} P_{b}^{+}+c_{-} P_{b}^{-}
$$

where $c_{+}$and $c_{-}$are the charge and discharge loss coefficients respectively, while $P_{b}^{+}$and $P_{b}^{-}$are the positive and negative parts of $P_{b}$ (i.e. positive variables with $P_{b}=P_{b}^{+}-P_{b}^{-}$). This is very popular when the energy management problem is expressed with a linear program (LP). Indeed, as said in Remark 2 above, rather than using the explicit expression (7) for $g$, it is better formulated as a LP:

$$
g=\min _{P_{b}^{+}, P_{b}^{-}} c_{+} P_{b}^{+}+c_{-} P_{b}^{-}
$$

with constraints on the variables so that, at the optimum, those are indeed the positive and negative parts of $P_{b}$ :

$$
\begin{aligned}
P_{b}^{+} & \geq P_{b} \\
P_{b}^{-} & \geq-P_{b} \\
P_{b}^{+}, P_{b}^{-} & \geq 0 \\
P_{b}^{+}, P_{b}^{-} & \leq P_{\text {rated }}^{+}, P_{\text {rated }}^{-}
\end{aligned}
$$

Using Remark 4, this loss formulation is more commonly injected directly in the storage dynamics (1) which becomes:

$$
E_{b}(k+1) \leq E_{b}(k)+\left(\eta_{+} P_{b}^{+}+P_{b}^{-} / \eta_{-}\right) \Delta_{t}
$$

Storage model (10) is obtained by replacing $P_{b}$ with $P_{b}^{+}-$ $P_{b}^{-}$and introducing the charging and discharging efficiency coefficients $\eta_{+}=1-c_{+}$and $\eta_{-}=1 /\left(1+c_{-}\right)$. Expression (10) is more compact than (1)+(7), but the transformations performed here show how it belongs to our generic framework of convex losses functions. Coefficients $\eta_{+}, \eta_{-}$can be chosen equal to the square root of the round-trip efficiency of a given storage technology. However, this does not make (7) a physical representation of losses, because a round-trip efficiency is valid for a given cycle only, since instantaneous losses are rarely piecewise linear.

In the literature, expression (10) is more often written with equality rather than $\leq$. An equality constraint is allowed here 


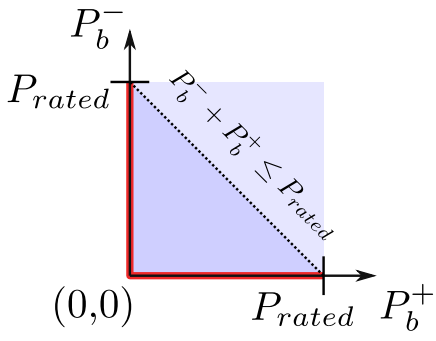

Fig. 2. Complementarity constraint (bold red lines) + convex relaxation (dark blue triangle), vs box constraint (light blue square)

because the expression is linear in $\left(P_{b}^{+}, P_{b}^{-}\right)$, but it is still a case of relaxed losses like (6), because the variables $P_{b}^{+}$and $P_{b}^{-}$have no complementarity constraint. This means that they can be both positive, with no effect on the net flow $P_{b}$, but which creates an artificial excess of losses.

Complementarity can be enforced by either a nonlinear complementarity constraint $P_{b}^{+} \cdot P_{b}^{-}=0$ [5] or by introducing a binary variable which creates a Mixed Integer LP (MILP) [16]. We do not detail these possibilities since they are both not convex. Only, we highlight that it is the exactness of this LP relaxation that was most thoroughly studied (no comparable results for nonlinear loss models). First, sufficient mathematical conditions have been identified for the relaxation to be tight [14], [15] (though we mentioned in §II-D that their applicability is limited). Second, the usage of an equality sign in (10) creates an upper bound for the worst-case amount of artificially wasted energy, when the relaxation fails. This worst-case bound (given in [5, App. A]) is better (lower) when the pair of constraints (9d) is replaced by the tighter limit

$$
P_{b}^{+} / P_{\text {rated }}^{+}+P_{b}^{-} / P_{\text {rated }}^{-} \leq 1
$$

which can be either viewed as the tightest convex approximation of the nonlinear complementarity constraint or derived by relaxing the integrality constraint in the MILP formulation. See Fig. 2 (draft).

\section{B. Quadratic-in-P Loss Model}

The second quite popular convex loss model is quadratic in power (detailed in [4], with a close variant in [10]):

$$
g\left(P_{b}, E_{b}\right)=\rho . P_{b}^{2}
$$

with positive parameter $\rho$. Compared to the PWL-in- $P$ model from section III-A, quadratic losses penalize more strongly large charge/discharge rates, while small rates are asymptotically lossless.

For optimization purpose, expression (12) is convex but nonlinear, so that a QP or a general Non-Linear Programming (NLP) solver must be used (or the quadratic function must be approximated by a set of a few tangents).

Physically, the loss model (12) mimics, inside an energybased model, the Joule losses on the series resistance of a circuit model (see Fig. 1). To understand this, we need to state the equivalence between the two. Conversion from circuit variables $i, v$ to power is

$$
P_{b}=v \cdot i=v_{0} \cdot i+r \cdot i^{2},
$$

where the last term is equal to the Joule losses. This expression can be solved for the current:

$$
i=\frac{P_{b}}{v_{0}} \times \frac{2}{1+\sqrt{1+\frac{4 r P_{b}}{v_{0}^{2}}}}
$$

which is defined for $P_{b} \geq-\frac{v_{0}^{2}}{4 r}$ (i.e. the circuit model embeds a physical discharge power limit). Expression (14) is the product of a term linear in $P_{b}$ and a nonlinear correction factor which tends to one for small power $\left(\left|P_{b}\right| \ll \frac{v_{0}^{2}}{4 r}\right.$, which is the same as assuming a small voltage drop: $\left.\left|v-v_{0}\right| \ll v_{0}\right)$. As a consequence, when substituting this expression of $i$ in the Joule losses, one gets an approximately quadratic expression

$$
P_{\text {loss }} \approx \frac{r}{v_{0}^{2}} P_{b}^{2}
$$

from which one can identify the physical definition of the quadratic loss coefficient $\rho$ :

$$
\rho=r / v_{0}^{2} .
$$

This analysis shows three conditions for the quadratic-in$P$ losses model to indeed match the Joule losses of a circuit model:

1) Charge/discharge rate must be small

2) Series resistance $r$ must be constant

3) Open circuit voltage (OCV) $v_{0}$ must be constant

The first condition can be lifted, if needed, because the loss expression without approximation is not quadratic but still convex (not shown here).

Lifting the "constant resistance \& OCV" conditions is the motivation for the model introduced in the next section.

\section{The "Convex Monomial Loss Model"}

Based on the physical limitations of the quadratic loss model we search for a loss expression which can depend on the State of Energy and be separable like (15). Also, the dependency in $P$ is assumed a power law $P_{b}^{a}$, so that our model embeds both PWL and quadratic-in- $P$ loss models with $a=1$ and $a=2$ respectively. The model would be $\rho\left(E_{b}\right) \times P_{b}^{a}$, but to handle both signs of $P_{b}$ when $a \neq 2$, we split the expression for the positive and negative parts of the power, like for the PWL-in- $P$ model in III-A. Finally, to enable an easier convexity analysis, we impose that the dependency in the SoE should also be a power law, albeit with a negative exponent (for convexity). Here is our proposed "convex monomial loss model":

$$
g\left(P_{b}, E_{b}\right)=c_{+} \frac{\left(P_{b}^{+}\right)^{a_{+}}}{\left|E_{b}-e_{+}\right|^{b_{+}}}+c_{-} \frac{\left(P_{b}^{-}\right)^{a_{-}}}{\left|E_{b}-e_{-}\right|^{b_{-}}}
$$

with power exponents $a_{+}, a_{-} \geq 1$ (likely equal), energy exponents $b_{+}, b_{-} \geq 0$, scaling parameters $c_{+}, c_{-} \geq 0$ (like in (7), but not with the same physical dimension) and parameters $e_{+}, e_{-}$which are the energy value for which losses tend to 
Lossless storage ( $\left.E_{\text {grid }} 1.000 \mathrm{kWh}, C_{\text {grid }} 0.2000 €\right)$

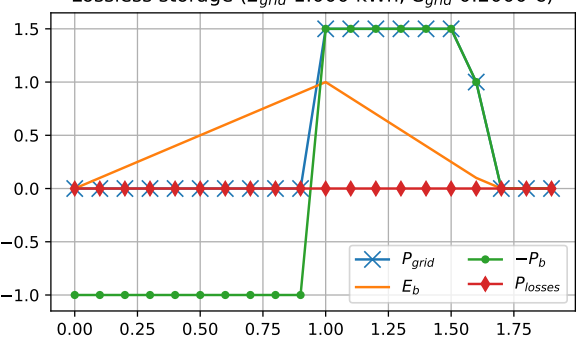

Quad-in- $P_{b}$ losses $\left(E_{\text {grid }} 0.800 \mathrm{kWh}, C_{\text {grid }} 0.1600 €\right)$

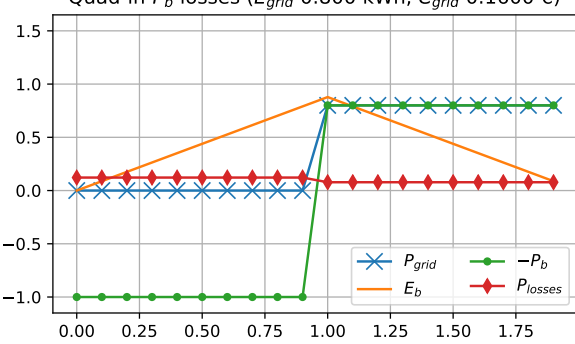

PWL-in- $P_{b}$ losses $\left(E_{\text {grid }} 0.800 \mathrm{kWh}, C_{\text {grid }} 0.1600 €\right)$

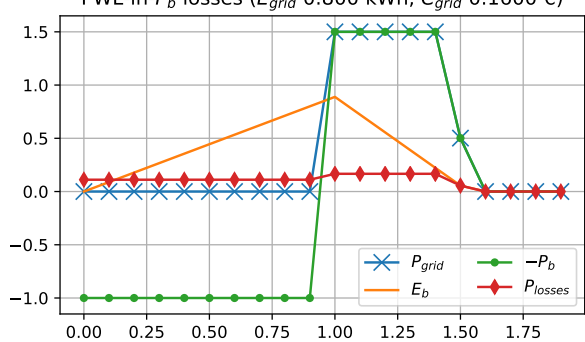

$\rho\left(E_{b}\right) . P_{b}^{2}$ losses $\left(E_{\text {grid }} 0.800 \mathrm{kWh}, C_{\text {grid }} 0.1599 €\right)$

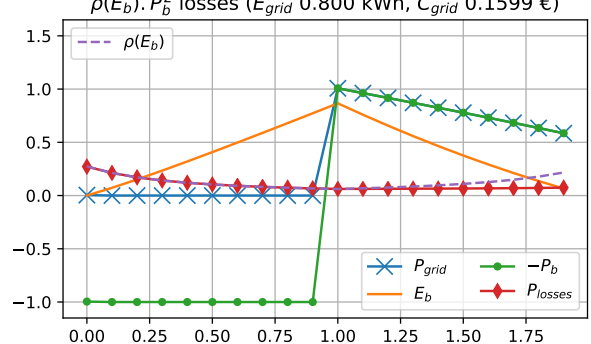

Linear-in- $E_{b}$ losses ( $\left.E_{\text {grid }} 0.800 \mathrm{kWh}, C_{g r d} 0.1599 €\right)$

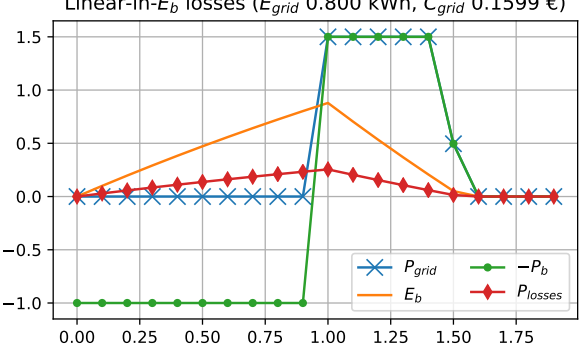

$\rho\left(E_{b}\right) . P_{b}^{2}$ losses $\left(E_{\text {grid }} 0.750 \mathrm{kWh}, C_{\text {grid }} 0.1465 €\right)$

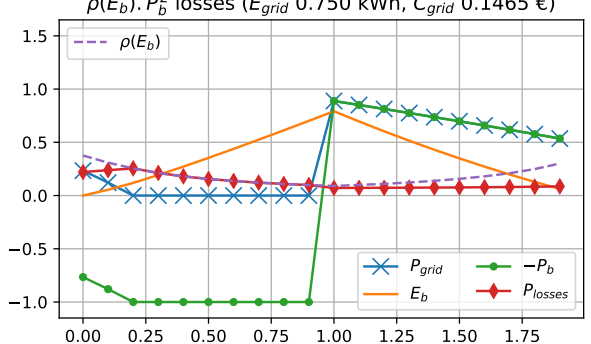

Fig. 3. Production shifting optimization for different storage loss models. Scenario is $1 \mathrm{~kW}$ production while low electricity price during $1^{\text {st }}$ hour and no production while high electricity price during $2^{\text {nd }}$ hour. All lossy models are approximately calibrated to show a $80 \%$ round trip storage efficiency, except the last one which is a variant at $75 \%$. With such efficiency, all models leverage the price difference by shifting the production to the $2^{\text {nd }}$ hour. However, the discharge profile varies with the loss model. Source: [17].

$+\infty$, so we must have $e_{+}, e_{-}<0$ or $>E_{\text {rated }}$. In case the behavior in charge and discharge is symmetrical, expression (17) simplifies to:

$$
g\left(P_{b}, E_{b}\right)=\underbrace{\frac{c}{\left|E_{b}-e\right|^{b}}}_{\rho\left(E_{b}\right)} \times\left|P_{b}\right|^{a}
$$

Example: For a capacitor $C$, we have a quadratic relation between energy and open circuit voltage: $E_{b}=C\left(v_{0}^{2}-v_{\varepsilon}^{2}\right) / 2$, where $v_{\varepsilon}$ is the lowest useful OCV value for which the capacitor is defined as empty (useable energy $E_{b}=0$ ). With it, the Joule losses expression (15) becomes $P_{\text {loss }} \approx \frac{r C / 2}{E_{b}+C v^{2} / 2} P_{b}^{2}$. Thus we identify the following coefficients in model (18): $a=2, b=1, c=r C / 2, e=-C v_{\varepsilon}^{2} / 2$. Notice that a direct derivation of this $P^{2} / E$ model was first proposed in [11].

\section{A. Convexity Conditions for the Monomial Loss Model}

We analyze the convexity of the first term of expression (17) since it is the sum of two similar expressions and since addition preserves convexity. We recognize that this term is the positive scaling of the 2D monomial $f_{a, b}: x, y \mapsto x^{a} y^{b}$, with arguments being $P_{b}^{+}$and an affine expression of $E_{b}$. Indeed the first term is $c_{+} \cdot f_{a_{+},-b_{+}}\left(P_{b}^{+}, \pm\left(E_{b}-e_{+}\right)\right)$. The \pm is " + " if $e_{+}<0$ and "-" if $e_{+}>E_{\text {rated }}$. This transformation preserves the convexity of $f$ (see $[1, \S 3.2 .2]$ ). We refer to the Appendix and Fig. 4 for the convexity analysis of $f_{a, b}$. (noticing that $f$ uses a flipped sign convention for exponent $b$ to make it symmetric between $a$ and $b$ ). The main result is that the loss model (17) is convex if:

$$
b_{+} \leq a_{+}-1, \quad b_{-} \leq a_{-}-1
$$

As an illustration, for the quadratic-in- $P$ case $\left(a_{ \pm}=2\right)$, we must have $b_{ \pm} \in[0,1]$. We can thus observe that the
$P^{2} / E$ loss model of a capacitor $\left(b_{ \pm}=1\right)$ is on the "edge of convexity". Also, the monomial model shows that making the loss coefficients $c_{ \pm}$in the PWL-in- $P$ model (7) depend on SoE (i.e. introduce an SoE dependent efficiency) would be not convex ( $a=1$ implies $b=0$ ).

\section{Illustration OF LOSS MODELS}

We now introduce an elementary energy storage application to show the effect of varied loss models on the optimal charge/discharge profile. One storage unit is used as a buffer between a power production $P_{\text {prod }}$ and a grid injection $P_{\text {grid }}$. Conservation of power is: $P_{\text {grid }}=P_{\text {prod }}-P_{b}$. The optimization objective is to maximize the profit from selling the production to the grid at a time varying price:

$$
\max C_{\text {grid }}=\sum_{k=1}^{K} c_{\text {grid }}(k) . P_{\text {grid }}(k) \Delta_{t}
$$

The price will be positive so that the loss relaxation always works (see "positive price argument" in §II-D). The scenario is a production shifting on two hours ( $K=20, \Delta_{t}=0.1 \mathrm{~h}$ ), with a $E_{\text {rated }}=1 \mathrm{kWh}$ storage. For the first hour, $P_{\text {prod }}=1 \mathrm{~kW}$ and price is low $\left(c_{\text {grid }}=0.1 € / \mathrm{kWh}\right)$. Then for the second hour, zero production and price is high $\left(c_{\text {grid }}=0.2 € / \mathrm{kWh}\right)$. The storage, described by (1) and varying loss models, can shift the production to the high price hour, if losses are small enough.

Optimal trajectories are shown on Fig. 3 for the lossless storage and four loss models: PWL-in- $P_{b}$ (7) $\left(c_{ \pm}=0.111\right)$, linear-in- $E_{b}(5) \quad\left(c_{E}=0.29 \mathrm{~W} / \mathrm{Wh}\right)$, quadratic-in- $P_{b}$ (12) $\left(\rho=0.122 \mathrm{~W} / \mathrm{W}^{2}\right)$ and the symmetric monomial loss model (18), tuned for a capacitor $(a=2, b=1, c=0.0685, e=$ $-0.25 \mathrm{kWh}$ ), that is losses are higher at low $\mathrm{SoE}$ for a given 
power. All models are tuned to yield an $80 \%$ round-trip efficiency on this scenario to make them comparable (except the last plot, see below). We checked that for all models the relaxed loss inequality (6) is tight, that is the relaxation works.

It can be observed that the charging phase is the same for all, with the storage absorbing all the production $\left(P_{b}=+1 \mathrm{~kW}\right)$. Then, despite the same round-trip efficiency, there is a great variety in discharge profiles, depending on the loss model: $\propto P_{b}^{2}$ losses smooth out the discharge on the entire second hour to reduce power peaks, while the lossless and PWL models allow faster discharge ${ }^{2}$ since efficiency is constant. The $\propto E_{b}$ losses push for the earliest discharge. Finally, the $\rho\left(E_{b}\right) P_{b}^{2}$ model shows a decaying discharge rate, to prevent loss increase at low SoE. That is $P_{\text {loss }}$ stays quite constant despite the rising loss coefficient $\rho\left(E_{b}\right)$.

The last plot of Fig. 3 is a variant of this model, with slightly higher losses $(c=0.094$, round-trip efficiency is $75 \%)$. This shows that the early charging is done at a reduced rate, with some of the production sold directly at a low price, because of the high initial value of $\rho$. Such time-varying partial arbitrage during a constant price period can only be obtained with an SoE-dependent loss model.

Optimization results were obtained by describing optimization problems in Julia using the JuMP package [18]. Solvers were Ipopt [19] and ECOS [20]. The complete code is available in a Jupyter notebook [17].

\section{CONCLUSION}

The relaxation of storage losses enable more realistic loss models while preserving convexity. We described this relaxation in a generic way, unifying the existing works which are often split by model types (e.g. piecewise linear vs. others), and we proposed one flexible model which contains all existing loss expressions.

Future work includes studying the case when the relaxation fails, e.g. generalizing the worst case bound which exists for the PWL-in- $P$ model. Also, including other variables influencing losses (temperature, aging), would be useful. Finally, fitting our model to actual battery loss data would confirm its usefulness.

\section{ACKNOWLEDGMENT}

We thank Olivier Mégel for a discussion back at PowerTech 2015 which planted the seed of our reflection on the convexity of losses. We thank Bertrand Cornélusse for providing us with useful references on relaxation in optimal power flow.

\section{APPENDIX}

\section{CONVEXITY OF THE LOSS HELPER FUNCTION}

We define the loss helper function as the $2 \mathrm{D}$ monomial

$$
f: x, y \mapsto x^{a} y^{b},
$$

\footnotetext{
${ }^{2}$ There is no unique solution to the optimization problem (20) with zero or PWL-in- $P$ losses, so we add a tiny increasing penalty coefficient to $P_{\text {grid }}$ to select the "earliest injection" solution among all optimal trajectories. This avoids getting a random one. A later discharge would be equally optimal.
}

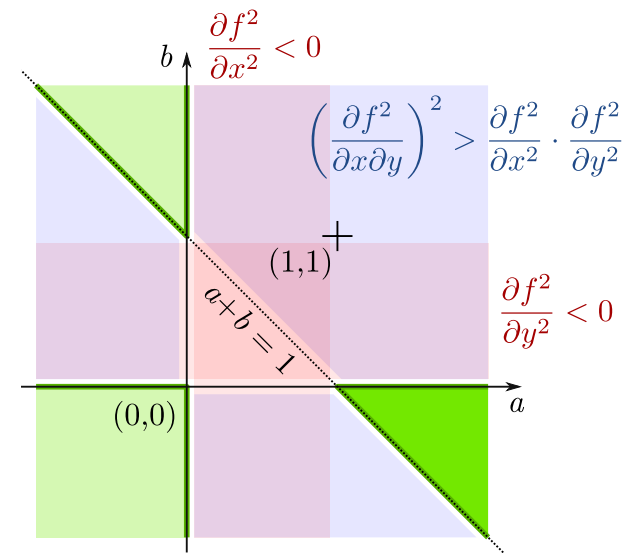

Fig. 4. Convexity of the losses helper function $f: x, y \mapsto x^{a} y^{b}$ on $\mathbb{R}_{+}^{2}$, depending on exponents $a$ and $b$. Red area $0<a<1$ is excluded because $f$ is concave in the $x$-direction (same for $b$ ). Blue regions are excluded because the determinant of the Hessian is negative. The three convex areas are in green. Source: [17].

defined for positive arguments $x, y$ and real exponents $a, b$.

The convexity of $f$ is equivalent to the positive definiteness of its Hessian:

$$
H(x, y)=\left(\begin{array}{cc}
a(a-1) x^{a-2} y^{b} & a b x^{a-1} y^{b-1} \\
a b x^{a-1} y^{b-1} & b(b-1) x^{a} y^{b-2}
\end{array}\right)
$$

$H$ is positive definite if and only if:

- each diagonal term is positive (i.e. $f$ is convex the $x$ and $y$ directions)

- the determinant is positive

The areas where these two conditions are not fulfilled are highlighted in red and blue respectively on the $(a, b)$ plane in Fig. 4. This leaves three convex regions in green. In this analysis, we assume that variable $x$ will be mapped to the storage power $P_{b}\left(\right.$ or $\left.-P_{b}\right)$. Since losses are generally increasing with the charging/discharging power, only the region with positive exponent $a$ makes physical sense. Thus the single region with interesting exponent values, highlighted on Fig. 4, is:

$$
\{(a, b) \text { such that } a+b \geq 1, b \leq 0\}
$$

\section{REFERENCES}

[1] S. Boyd and L. Vandenberghe, Convex Optimization. Cambridge University Press, 2004.

[2] X. Chen, E. Dall'Anese, C. Zhao, and N. Li, "Aggregate Power Flexibility in Unbalanced Distribution Systems," IEEE Transactions on Smart Grid, vol. 11, no. 1, pp. 258-269, Jan 2020.

[3] Q. Gemine, D. Ernst, and B. Cornélusse, "Active network management for electrical distribution systems: problem formulation, benchmark, and approximate solution," Optimization and Engineering, vol. 18, no. 3, pp. 587-629, 2017.

[4] N. Murgovski, L. Johannesson, J. Sjöberg, and B. Egardt, "Component sizing of a plug-in hybrid electric powertrain via convex optimization," Mechatronics, vol. 22, no. 1, pp. 106 - 120, 2012.

[5] M. R. Almassalkhi and I. A. Hiskens, "Model-Predictive Cascade Mitigation in Electric Power Systems With Storage and Renewables-Part I: Theory and Implementation," IEEE Transactions on Power Systems, vol. 30, no. 1, pp. 67-77, Jan. 2015.

[6] S. Gill, I. Kockar, and G. W. Ault, "Dynamic Optimal Power Flow for Active Distribution Networks," IEEE Transactions on Power Systems, vol. 29, no. 1, pp. 121-131, Jan 2014 
[7] X. Wu, X. Hu, Y. Teng, S. Qian, and R. Cheng, "Optimal integration of a hybrid solar-battery power source into smart home nanogrid with plug-in electric vehicle," Journal of Power Sources, vol. 363, pp. 277 $283,2017$.

[8] O. Mégel, J. L. Mathieu, and G. Andersson, "Hybrid stochasticdeterministic multiperiod dc optimal power flow," IEEE Transactions on Power Systems, vol. 32, no. 5, pp. 3934-3945, Sep. 2017.

[9] A. Gonzalez-Castellanos, D. Pozo, and A. Bischi, "Detailed li-ion battery characterization model for economic operation," International Journal of Electrical Power \& Energy Systems, vol. 116, p. 105561, 2020.

[10] M. Nick, R. Cherkaoui, and M. Paolone, "Optimal allocation of dispersed energy storage systems in active distribution networks for energy balance and grid support," IEEE Transactions on Power Systems, vol. 29 , no. 5, pp. 2300-2310, Sep. 2014.

[11] N. Murgovski, L. Johannesson, and J. Sjöberg, "Convex modeling of energy buffers in power control applications," IFAC Proceedings Volumes, vol. 45, no. 30, pp. 92 - 99, 2012, 3rd IFAC Workshop on Engine and Powertrain Control, Simulation and Modeling.

[12] C. Pinto, J. V. Barreras, E. Schaltz, and R. E. Araújo, "Evaluation of advanced control for li-ion battery balancing systems using convex optimization," IEEE Transactions on Sustainable Energy, vol. 7, no. 4, pp. 1703-1717, Oct 2016.

[13] B. Li, R. Roche, and A. Miraoui, "Microgrid sizing with combined evolutionary algorithm and milp unit commitment," Applied Energy, vol. 188 , pp. $547-562,2017$.

[14] Z. Li, Q. Guo, H. Sun, and J. Wang, "Extended sufficient conditions for exact relaxation of the complementarity constraints in storage-concerned economic dispatch," CSEE Journal of Power and Energy Systems, vol. 4, no. 4, pp. 504-512, Dec 2018.

[15] C. Duan, L. Jiang, W. Fang, X. Wen, and J. Liu, "Improved sufficient conditions for exact convex relaxation of storage-concerned ed," 2016. [Online]. Available: https://arxiv.org/abs/1603.07875

[16] J. M. Arroyo, L. Baringo, A. Baringo, R. Bolaños, N. Alguacil, and N. G. Cobos, "On the use of a convex model for bulk storage in mip-based power system operation and planning," IEEE Transactions on Power Systems, vol. 35, no. 6, pp. 4964-4967, Nov 2020.

[17] P. Haessig, "pierre-haessig/convex-storage-loss v1.1.0," Nov. 2020. [Online]. Available: http://doi.org/10.5281/zenodo.4289203

[18] I. Dunning, J. Huchette, and M. Lubin, "JuMP: A Modeling Language for Mathematical Optimization," SIAM Review, vol. 59, no. 2, pp. 295320, 2017.

[19] A. Wächter and L. T. Biegler, "On the implementation of an interiorpoint filter line-search algorithm for large-scale nonlinear programming," Mathematical Programming, vol. 106, no. 1, pp. 25-57, 2006.

[20] A. Domahidi, E. Chu, and S. Boyd, "ECOS: An SOCP solver for embedded systems," in 2013 European Control Conference (ECC), July 2013, pp. 3071-3076. 